\title{
Birds of Parque Estadual do Tainhas, an important protected area of the highland grasslands of Rio Grande do Sul, Brazil
}

\author{
Eduardo Chiarani ${ }^{1,2}$ \& Carla Suertegaray Fontana ${ }^{1,3}$ \\ 1 Pontifícia Universidade Católica do Rio Grande do Sul (PUC-RS), Escola de Ciências, Programa de Pós-Graduação em Ecologia e Evolução da \\ Biodiversidade (PPGEEB), Museu de Ciências e Tecnologia (MCT), Laboratório de Ornitologia. Porto Alegre, RS. Brasil. \\ 2 ORCID: http://orcid.org/0000-0002-4673-6473. E-mail: educhiarani@gmail.com \\ 3 ORCID: http://orcid.org/0000-0001-9423-0752. E-mail: carla@pucrs.br
}

\begin{abstract}
Parque Estadual do Tainhas - PET (Tainhas State Park) is a protected area with significant extensions of natural grasslands located in highland grasslands of northeastern Rio Grande do Sul state, Brazil, and recognized as an Important Bird Area. Our aim was to record the bird species richness of PET with a greater sampling effort than previous studies at the site, including unpublished data such as occurrence status of most species and evidence of breeding of some of them in the park. We surveyed birds between 2012 and 2018 performing aleatory trails covering mainly the central portion of PET (1,500 ha). We also added records from published studies. We listed 208 species, of which 203 were recorded during our fieldwork (including 18 species of conservation concern). The estimated richness was 222.22 (SD = 10.91) species, according to Jackknife 1 method. This result represents a $58 \%$ increase in the species richness of the park and shows that long-term monitoring of the bird community allows to access more accurate richness and to identify the regular species (core avifauna) of the park. The presence of 55 breeders and threatened species shows the importance of this protected area for breeding and conservation of grassland birds, such as Urubitinga coronata, Cinclodes pabsti, Xolmis dominicanus, Anthus nattereri, Xanthopsar flavus, and Sporophila melanogaster.
\end{abstract}

Key-Words. Breeding; Campos de Cima da Serra; Conservation unit; Grassland birds; Species richness.

\section{INTRODUCTION}

Grasslands are the most representative vegetation type in extreme southern Brazil (Andrade et al., 2019). Despite this, it is one of the most neglected ecosystems considering that few grassland areas are protected in conservation units of integral protection in Brazil (less than 0.5\%) (Overbeck et al., 2007; Pillar \& Vélez, 2010). Approximately $25 \%$ of the grasslands in southern Brazil have been lost in the last three decades (Overbeck et al., 2007), mainly by changes in land use that affect grasslands over the world, such as expansion of crops, forestation with exotic species, and pastures with exotic grasses for livestock (Gibson, 2009; Bond \& Parr, 2010). Brazilian conservation policies are strongly focused on forest biomes (e.g., Amazon and Atlantic Forest), while little importance is given to non-forest ecosystems (Overbeck et al., 2015). Therefore, knowledge on the diversity of these open habitats is a basic information necessary to expand the protected areas and contribute to a suitable management of current grassland conservation units.
In the Atlantic Forest biome the grassland landscape forms a mosaic with forests, known as Campos do Planalto Meridional or Campos de Altitude (highland grasslands) (MMA, 2000; IBGE, 2004). Specifically in northeastern Rio Grande do Sul and southeastern Santa Catarina states, this region receives the physiographic name of Campos de Cima da Serra due to the natural occurrence of grasslands in hills around $800 \mathrm{~m}$ above sea level (Bond-Buckup \& Dreier, 2008). Campos de Cima da Serra show a complex natural physiognomy, with large extensions of grasslands interspersed with araucaria forests (Araucaria angustifolia), water courses, marshes and peat bogs (Hasenack \& Lucatelli, 2008; Boldrini, 2009). As in most of the grasslands in South America, the habitats of this region have been threatened by anthropogenic actions, such as afforestation with Pinus spp. and other tree species, crops, unmanaged fires, drainage of wetlands, and installation of hydroelectric dams (Fontana et al., 2003; Buckup \& Bond-Buckup, 2008). Although they are part of the Atlantic Forest biome, highland grasslands of southern Brazil show many affinities with large open biomes of 
South America in terms of avifauna similarities (Stotz et al., 1996; Sick, 1997), especially with the Pampa biome in Brazil (Fontana et al., 2008a). However, perhaps because it is inserted in the Atlantic Forest biome, Campos de Cima da Serra have been neglected in widely disseminated studies on open ecosystems (Fontana et al., 2008a).

Studies developed in Campos de Cima da Serra region have counted 1,161 plant species, of which 107 are endemic (Boldrini, 2009), and a high richness of birds (337 species), with several rare, endemic, and threatened species (Fontana et al., 2008a). Approximately $42 \%$ of these birds are associated with grassland habitats or marshes, while the others are associated with forest habitats (e.g., riparian forests and wood patches) (Fontana et al., 2008a, b). The Long-tailed Cinclodes (Cinclodes pabsti) is an endemic species of the southern Brazilian highland grasslands, occurring only in Campos de Cima da Serra region (Freitas et al., 2008). The region is extremally important for the Black-bellied Seedeater (Sporophila melanogaster) breeding, since this endemic species of Brazil breeds only in highland grasslands of Rio Grande do Sul and Santa Catarina (Bencke et al., 2003; Rovedder \& Fontana, 2012). One study carried out in two protected areas (Parque Nacional de Aparados da Serra and Parque Nacional da Serra Geral) with large extensions of grasslands in the region (sampled together) indicates the presence of 301 bird species (IBAMA, 2004).

Parque Estadual do Tainhas (Tainhas State Park - hereafter PET) is a protected area (a conservation unit) with 6,654 ha located in Campos de Cima da Serra region, covering portions of Jaquirana, São Francisco de Paula, and Cambará do Sul municipalities (Bencke \& Duarte, 2008). It is one of the few conservation units of integral protection in Rio Grande do Sul that includes significant extensions of natural grasslands (Bencke \& Duarte, 2008), and is also recognized as an Important Bird Area (IBA) due to its role in the conservation of threatened birds (Bencke et al., 2006). The complete implementation of PET and the expansion of its limits are recommended actions in a national plan for the conservation of birds in the grasslands of southern Brazil (Serafini, 2013). The terrestrial fauna of PET has a great representativeness of animals of open areas and associated habitats, such as marshes and peat bogs (Bencke \& Duarte, 2008). Several threatened species are found in the PET, mainly birds (Fontana et al., 2003; Bencke et al., 2006; Bencke \& Duarte, 2008; Serafini, 2013) and mammals (Bencke \& Duarte, 2008). The management plan of the park reported 132 bird species with confirmed occurrence in the area, plus 15 species of potential occurrence that occur in its immediate surroundings or in nearby areas with similar characteristics (Bencke \& Duarte, 2008). However, the sample effort used in this survey (from September $17^{\text {th }}$ to September $19^{\text {th }}$ and from October $17^{\text {th }}$ to October 19th, 2007 ; G.A. Bencke, pers. comm.) was small, suggesting that the bird richness of the park can be larger.

Our aim was to survey the bird species richness of Parque Estadual do Tainhas, an important protected area of the highland grasslands in southern Brazil, with a greater sampling effort than previous studies performed at the site. We also present unpublished data on the occurrence status of most species and breeding evidences of some species in the park, besides providing additional information on species of conservation concern.

\section{MATERIAL AND METHODS}

\section{Study area}

The study was performed in Parque Estadual do Tainhas, located in northeastern Rio Grande do Sul state, Brazil (Fig. 1). Sampling was carried out mainly in the central portion of PET, in an area of approximately 1,500 ha (nearly $1 / 4$ of the total area of the park), locat-

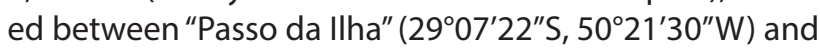
"Passo do S" (29 $\left.05^{\prime} 05^{\prime \prime} \mathrm{S}, 50^{\circ} 22^{\prime} 03^{\prime \prime} \mathrm{W}\right)$. Other areas were sampled non-systematically, such as a large forest patch in the northern part of PET sampled only once (in 2018) to access the possible presence of species not recorded before. Most of the area of the park is not implemented yet, and owners remain raising cattle and growing crops, besides managing grasslands with fire according to the established culture in the region. There are two main grassland vegetation management types in the sampled area of the park: (1) area burned only once in the last ten years (about 300 ha burned in 2016) and (2) areas burned annually or every two years for cattle grazing.

Vegetation types and environments found at PET are grasslands, marshes, peat bogs, rocky outcrops, and araucaria forests (Bencke \& Duarte, 2008) (Fig. 2). Grassland areas occupy most of the park, while native forest areas encompass only patches of araucaria and riparian forests of the Tainhas river and its tributaries. The climate of the region corresponds to the mesothermic humid (Vieira, 1984) or humid temperate (Maluf, 1999), and the annual mean temperature is $15.7^{\circ} \mathrm{C}$, with mean of $11.1^{\circ} \mathrm{C}$ for minimum temperatures and mean of $21.6^{\circ} \mathrm{C}$ for maximum temperatures (Rambo, 2000). The precipitation is well distributed over the year (1,500-1,700 mm annual mean), reaching values up to $2,500 \mathrm{~mm}$ in certain sub regions (Almeida, 2009).

\section{Data collection and analysis}

We collected data between 2012 and 2018, while carrying on other studies. Data are related to five breeding seasons (October to March). In four of them (2012-2013, 2013-2014, 2016-2017, and 2017-2018) one researcher (EC) stayed in the field full time (i.e., five months per season). In 2015-2016 sampling was carried out on a nonfull-time basis, totaling 30 days of fieldwork over the breeding season. There was no sampling in winter.

Birds were sampled qualitatively through daily random displacements in several habitats. Birds heard and/or visualized during these displacements were identified and recorded with the help of binoculars, digital camera and/ or audio recorder. Documented records (photographs and voices) were deposited on WikiAves website (www. 

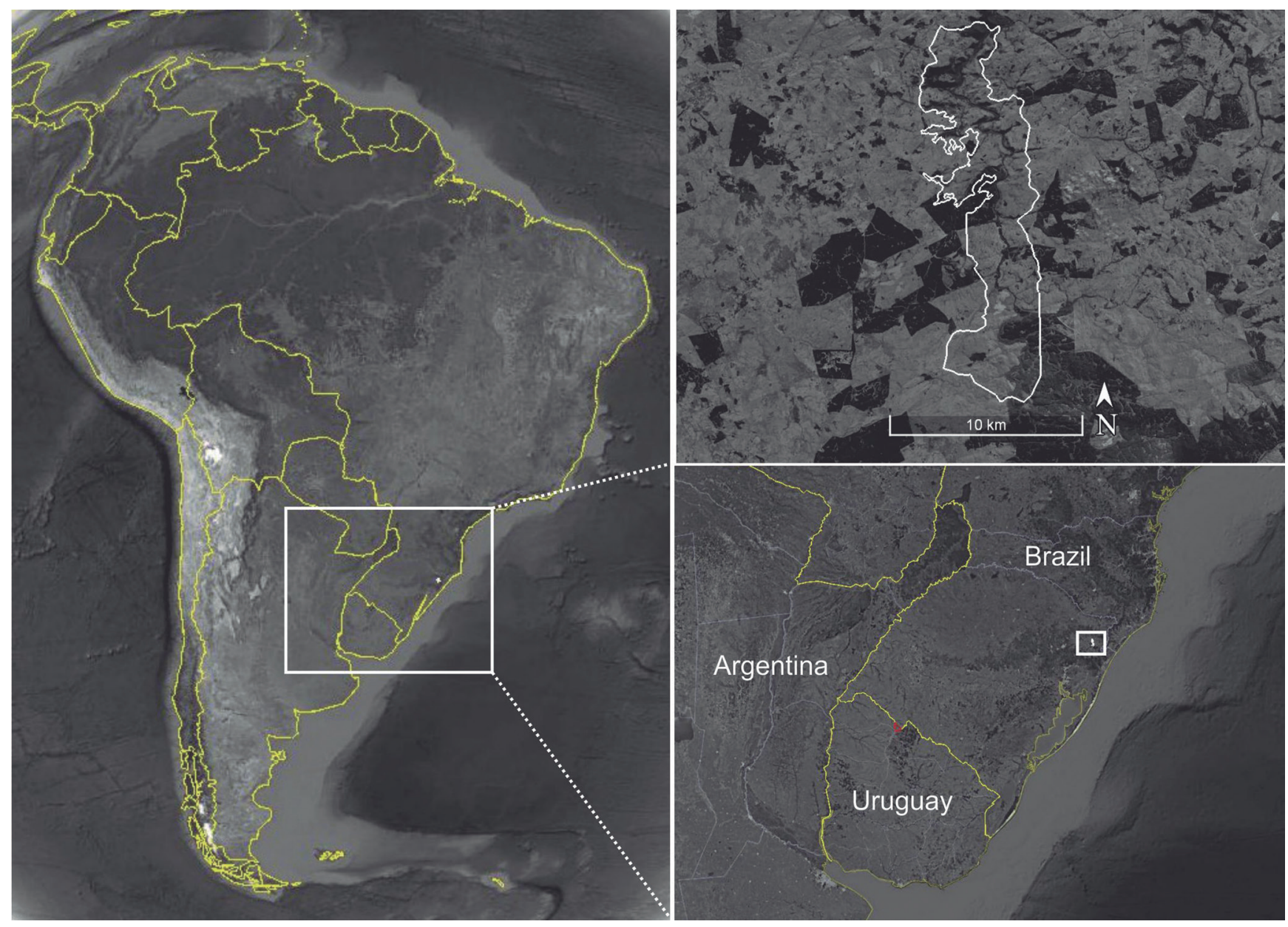

Figure 1. Location of Parque Estadual do Tainhas (white polygon on upper right detail) in southern Brazil. Source: Google Earth.

wikiaves.com). In addition, we included species records made in the park that are published in its management plan (Bencke \& Duarte, 2008) but that were not recorded in our field surveys. We also searched for species records deposited in online databases (Macaulay Library - www. macaulaylibrary.org; Xeno-canto - www.xeno-canto. org; and WikiAves) and specimens deposited in Brazilian museums (Museu de Ciências e Tecnologia, of Pontifícia Universidade Católica do Rio Grande do Sul, and Museu de Ciências Naturais, of Fundação Zoobotânica do Rio Grande do Sul) and North American collections (ORNIS digital database - www.ornisnet.org), whose locality record could be safely attributed to the PET or its vicinities.

In order to estimate the bird richness of PET we used the first-order Jackknife species richness estimator, since our data were based on incidence of the species (Gotelli \& Colwell, 2010). We also built a rarefaction curve with the observed richness, considering each breeding season as a sampling unit (sample-based rarefaction curve; Gotelli \& Colwell, 2010). This analysis was performed in EstimateS 9.1.0 (Colwell, 2013).

The confirmation of breeding activity of the species was made through observation of active nests under construction, with eggs or nestlings, or through observation of young individuals with limited flying ability ("prejuvenal") or smaller than adults (Binford, 1973; Maurício et al., 2013). For species whose juveniles have greater dispersion capacity (e.g., birds of prey) and did not have their nests found (only juveniles with parents), we considered that the breeding area can be the PET or its vicinities, since the immature with fully-grown flight feathers are capable of traveling long distances from the nest site (Binford, 1973). The status of occurrence of species in the PET was determined using the incidence values. Thus, we consider "regular" those species recorded in all the five breeding seasons sampled, usually more than five times per season; "occasional" those species recorded in two to four breeding seasons, usually with few records per season (from one to five times); and "rare" the species found few times (one to five) only in a single season. Regular species include migratory species, what does not mean these species may be found over the year in the park. The term "core avifauna" (sensu Remsen, 1994) can be applied to regular species (i.e., those species that regularly breed, winter, or migrate through the site). Species recorded only in the large forest fragment in the northern of PET, sampled only once, did not have their occurrence status verified.

The status of threat in state, country, and global levels follow Rio Grande do Sul (2014), MMA (2014), and IUCN (2018), respectively. Endemic species of the Atlantic Forest biome follow Bencke et al. (2006). Species restricted to grassland habitats or which make extensive use of grassland follow Azpiroz et al. (2012). Migratory species were classified according to Bencke (2001). Scientific names and the taxonomic order of the species follow Piacentini et al. (2015). 

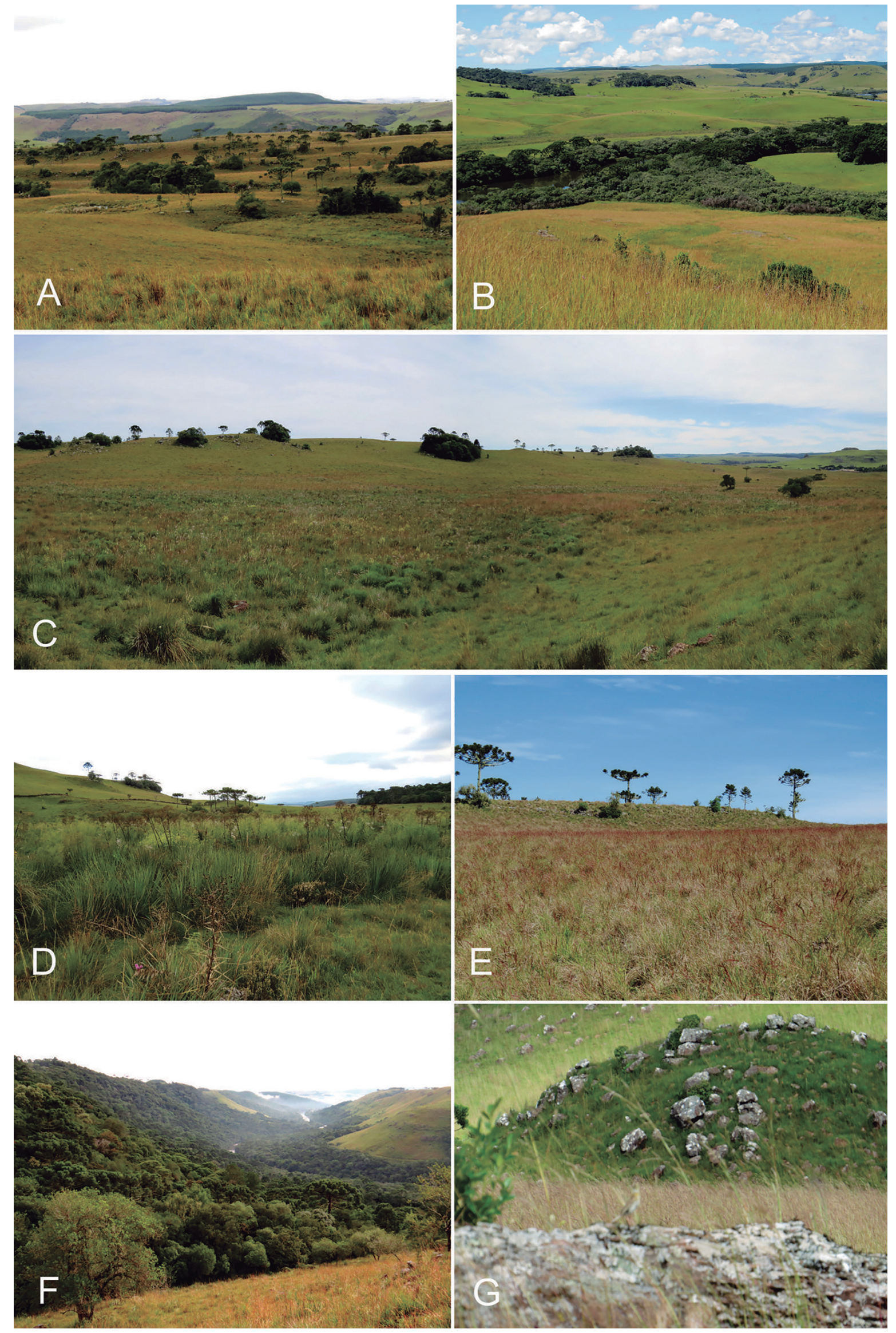

Figure 2. Habitats and vegetation types found in Parque Estadual do Tainhas: (A) landscape of PET showing grasslands interspersed with forest patches composed mainly of araucaria (Araucaria angustifolia), (B) riparian forest along the Tainhas river and its tributary, (C) grassland with marsh in lower areas, (D) marsh dominated by "gravatá" (Eryngium pandanifolium), (E) dry grassland composed mainly of Sorghastrum sp., (F) large forest area in the northern portion of PET, (G) rocky outcrop. Photos: Eduardo Chiarani. 


\section{RESULTS}

\section{Species list}

We recorded 203 bird species in Parque Estadual do Tainhas during fieldwork, of which 150 were documented (Table 1). Five species reported in the PET's management plan were not recorded during our study: Podilymbus podiceps, Sarcoramphus papa, Porphyriops melanops, Stephanoxis loddigesii, and Jacana jacana. We recorded $P$. melanops only outside PET's limits (about $2 \mathrm{~km}$ far from the East limit). Species records in online databases or specimens deposited in museums reported to the PET or nearby places (e.g., Várzea do Cedro, Lajeado Grande localities) do not represent additions to the list. Thus, the total number of bird species with confirmed occurrence inside PET's limits is currently 208 species. Species richness was estimated at $222.22(S D=10.91)$ species. Although the species accumulation curve does not show a clear trend towards stabilization, the observed richness corresponds to $91.4 \%$ of the estimated richness and is within its $95 \%$ confidence interval (Fig. 3 ).

Ten species are threatened, and eight are near-threatened in regional, national and/or global levels (described below). Migratory species correspond to $15 \%(n=31)$, while species restricted to grassland habitats totaled $24 \%$ ( $n=49$ ) of the birds of PET. Twenty-five species are endemic of the Atlantic Forest biome. Of the 198 species whose occurrence status was possible to determine, 146 (74\%) may be considered of regular presence (the "core avifauna"). Other 33 species (17\%) are occasional and 19 (9\%) were classified as locally rare.

\section{Species of conservation concern}

Ten species recorded are threatened in regional (RS), national (BR), and/or global (GL) levels, while other eight species are considered near threatened. Most of them are regular species in the PET, except Sporophila beltoni and S. hypoxantha, which are occasional in the area, and Sarcoramphus papa, which has not been evaluated (see below)

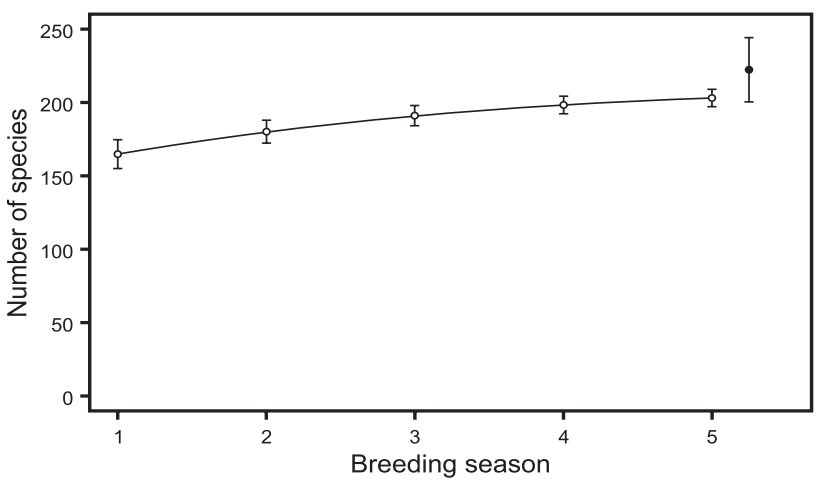

Figure 3. Rarefaction curve of bird species recorded over five breeding seasons (white dots) and estimated richness (black dot) in the Parque Estadual do Tainhas, Rio Grande do Sul, Brazil. Whiskers represent the $95 \%$ confidence interval.
Urubitinga coronata (Critically Endangered - RS; Endangered - BR, GL): at least a pair and two juveniles were recorded in the sampled area (see details in breeding data). The species was observed carrying a Southern LongNosed Armadillo (Dasypus hybridus) in flight (on October $22^{\text {nd }}, 2016$ ) and an unidentified snake (on December $2^{\text {nd }}$, 2012). This armadillo species was easily sighted in the area during our displacements, where it seems to be abundant.

Gallinago undulata (Vulnerable-RS): recorded with some regularity in larger marshes (at least in four marshes in the park), mainly through its voice at dusk. Probably its density is low (perhaps a single pair in each one of these marshes) making it a frequent albeit scarce species.

Amazona pretrei (Vulnerable - RS, BR, GL): couples or small groups of up to 12 individuals were observed with frequency flying over grassland areas to move among forest patches.

Cinclodes pabsti (Vulnerable - RS): found mainly close to rocky outcrops, roadsides and human constructions within the park. Family groups of up to five individuals were observed near "Passo do S", at the headquarters of the park.

Xolmis dominicanus (Vulnerable - RS, GL): common in the park, but apparently occurring in low density. Recorded almost always in pairs and only in some marshes of the park, often together with Xanthopsar flavus, another threatened species (see below).

Anthus nattereri (Vulnerable - RS, BR, GL): frequently recorded in the park, where it seems to be more abundant in areas burned periodically. In the area without regular fire few territories were observed, usually on hill tops where the grass is lower.

Xanthopsar flavus (Vulnerable - RS, BR, GL): common in the park, found usually in groups in the marshes or foraging in the grasslands around them, usually together with $X$. dominicanus. The largest recorded flock contained 52 individuals and was observed in a marsh surrounded by burned grassland near "Passo do S" (on September 28 ${ }^{\text {th }}, 2015$ ).

Sporophila hypoxantha (Vulnerable - RS, BR): in three years (2012, 2015 and 2016) only one adult male was occasionally observed per season. In 2015 we banded one male which returned to the same territory in 2016. In the next season (2017-2018) the male did not return, which indicates that the occurrence of the species in the park may be occasional.

Sporophila melanogaster (Endangered - RS; Vulnerable $\mathrm{BR})$ : this species is abundant in the marshes of PET and may be found with facility from November to March, when it breeds (see details in breeding data).

Sporophila beltoni (Endangered - RS; Vulnerable - BR): a single adult male was observed only for a few minutes in December 2015 and was not seen again in the same 
Table 1. List of bird species recorded in Parque Estadual do Tainhas (PET), northeastern Rio Grande do Sul state, Brazil. Species marked with asterisk indicate records made only in the management plan of the park. 0 ccurrence status: $R=$ Regular, $0=0$ ccasional, and $R a=$ Rare. Conservation status in regional (RS), national $(B R)$, and global (GL) levels: $(R=$ Critically endangered, $E N=$ Endangered, $V U=$ Vulnerable, and $N T=$ Near-threatened. Breeding evidence: $N=$ Nest (active), $P=$ Prejuvenal with limited flying ability or smaller than adults, and $\mathrm{J}=$ Juveniles with fully-grown flight feathers, seen together with adult individual. Voucher number from records deposited on WikiAves website can be accessed online using http://www.wikiaves.com/"voucher number".

\begin{tabular}{|c|c|c|c|c|}
\hline \multirow{2}{*}{ Taxa } & \multicolumn{2}{|c|}{ Status } & \multirow{2}{*}{ Breeding evidence } & \multirow{2}{*}{ Voucher numbe } \\
\hline & Occurrence in PET & Conservation & & \\
\hline \multicolumn{5}{|l|}{ Tinamidae } \\
\hline Crypturellus obsoletus (Temminck, 1815) & $\mathrm{R}$ & & & \\
\hline Rhynchotus rufescens (Temminck, 1815) & $\mathrm{R}$ & & $\mathrm{N}, \mathrm{P}$ & 1198695 \\
\hline Nothura maculosa (Temminck, 1815) & $\mathrm{R}$ & & $\mathrm{N}$ & 3197749 \\
\hline \multicolumn{5}{|l|}{ Anhimidae } \\
\hline Chauna torquata (0ken, 1816) & Ra & & & 3186136 \\
\hline \multicolumn{5}{|l|}{ Anatidae } \\
\hline Amazonetta brasiliensis (Gmelin, 1789) & $\mathrm{R}$ & & & 3186169 \\
\hline Anas flavirostris Vieillot, 1816 & $\mathrm{R}$ & & $p$ & 747850 \\
\hline Anas georgica Gmelin, 1789 & 0 & & & 3186165 \\
\hline \multicolumn{5}{|l|}{ Cracidae } \\
\hline Penelope obscura Temminck, 1815 & $\mathrm{R}$ & & & 3189200 \\
\hline \multicolumn{5}{|l|}{ Podicipedidae } \\
\hline \multicolumn{5}{|l|}{ Podilymbus podiceps (Linnaeus, 1758)* } \\
\hline \multicolumn{5}{|l|}{ Ciconiidae } \\
\hline Ciconia maguari (Gmelin, 1789) & R & & & 3185179 \\
\hline Mycteria americana Linnaeus, 1758 & 0 & & & 3185177 \\
\hline \multicolumn{5}{|l|}{ Phalacrocoracidae } \\
\hline Phalacrocorax brasilianus (Gmelin, 1789) & $\mathrm{R}$ & & & \\
\hline \multicolumn{5}{|l|}{ Ardeidae } \\
\hline Nycticorax nycticorax (Linnaeus, 1758) & $\mathrm{R}$ & & & \\
\hline Butorides striata (Linnaeus, 1758) & $\mathrm{R}$ & & & 1211733 \\
\hline Bubulcus ibis (Linnaeus, 1758) & $\mathrm{R}$ & & & \\
\hline Ardea cocoi Linnaeus, 1766 & 0 & & & \\
\hline Ardea alba Linnaeus, 1758 & R & & & \\
\hline Syrigma sibilatrix (Temminck, 1824) & $\mathrm{R}$ & & & 3187204 \\
\hline Egretta thula (Molina, 1782) & $\mathrm{R}$ & & & \\
\hline \multicolumn{5}{|l|}{ Threskiornithidae } \\
\hline Plegadis chihi (Vieillot, 1817) & Ra & & & 3187205 \\
\hline Phimosus infuscatus (Lichtenstein, 1823) & Ra & & & 3187226 \\
\hline Mesembrinibis cayennensis (Gmelin, 1789) & $\mathrm{R}$ & NT (RS) & & 876244 \\
\hline Theristicus caudatus (Boddaert, 1783) & R & & & 1204046 \\
\hline \multicolumn{5}{|l|}{ Cathartidae } \\
\hline Cathartes aura (Linnaeus, 1758) & $\mathrm{R}$ & & & 3186161 \\
\hline Coragyps atratus (Bechstein, 1793) & R & & & 3187228 \\
\hline Sarcoramphus papa (Linnaeus, 1758)* & & NT (RS) & & \\
\hline \multicolumn{5}{|l|}{ Accipitridae } \\
\hline Elanus leucurus (Vieillot, 1818) & R & & & 3186134 \\
\hline Circus buffoni (Gmelin, 1788) & 0 & & & 3186137 \\
\hline Accipiter striatus Vieillot, 1808 & R & & & \\
\hline Accipiter bicolor (Vieillot, 1817) & Ra & & & 3042081 \\
\hline Rostrhamus sociabilis (Vieillot, 1817) & Ra & & & 3186159 \\
\hline Geranospiza caerulescens (Vieillot, 1817) & Ra & & & 3038041 \\
\hline Heterospizias meridionalis (Latham, 1790) & R & & J & 714485 \\
\hline Urubitinga urubitinga (Gmelin, 1788) & Ra & & & \\
\hline Urubitinga coronata (Vieillot, 1817) & R & $\mathrm{CR}(\mathrm{RS}) ; \mathrm{EN}(\mathrm{BR}, \mathrm{GL})$ & J & 876258 \\
\hline Rupornis magnirostris (Gmelin, 1788) & R & & & \\
\hline Geranoaetus albicaudatus (Vieillot, 1816) & $\mathrm{R}$ & & $\mathrm{J}$ & 1204037 \\
\hline Geranoaetus melanoleucus (Vieillot, 1819) & $\mathrm{R}$ & NT (RS) & J & 876265 \\
\hline Aramidae & & & & \\
\hline Aramus guarauna (Linnaeus, 1766) & 0 & & & 3191672 \\
\hline rat & & & & \\
\hline Aramides saracura (Spix, 1825) & R & & $P$ & 3187236 \\
\hline Laterallus leucopyrrhus (Vieillot, 1819) & $\mathrm{R}$ & & & 2484589 \\
\hline Pardirallus nigricans (Vieillot, 1819) & 0 & & & \\
\hline Pardirallus sanguinolentus (Swainson, 1837) & $\mathrm{R}$ & & $p$ & 3187237 \\
\hline Gallinula galeata (Lichtenstein, 1818) & $\mathrm{R}$ & & & \\
\hline Porphyriops melanops (Vieillot, 1819)* & & & & \\
\hline Charadriidae & & & & \\
\hline Vanellus chilensis (Molina, 1782) & $\mathrm{R}$ & & $\mathrm{N}, \mathrm{P}$ & 3187256 \\
\hline
\end{tabular}




\begin{tabular}{|c|c|c|c|c|}
\hline \multirow{2}{*}{ Taxa } & \multicolumn{2}{|c|}{ Status } & \multirow{2}{*}{ Breeding evidence } & \multirow{2}{*}{ Voucher number } \\
\hline & Occurrence in PET & Conservation & & \\
\hline \multicolumn{5}{|l|}{ Recurvirostridae } \\
\hline Himantopus melanurus Vieillot, 1817 & 0 & & & 2902509 \\
\hline \multicolumn{5}{|l|}{ Scolopacidae } \\
\hline Gallinago paraguaiae (Vieillot, 1816) & $\mathrm{R}$ & & $\mathrm{N}$ & 3197772 \\
\hline Gallinago undulata (Boddaert, 1783) & $\mathrm{R}$ & VU (RS) & & 3198855 \\
\hline Actitis macularius (Linnaeus, 1766) & 0 & & & 3038022 \\
\hline \multicolumn{5}{|l|}{ Jacanidae } \\
\hline \multicolumn{5}{|l|}{ Jacana jacana (Linnaeus, 1766)* } \\
\hline \multicolumn{5}{|l|}{ Columbidae } \\
\hline Columbina talpacoti (Temminck, 1810) & Ra & & & 3186128 \\
\hline Patagioenas picazuro (Temminck, 1813) & $\mathrm{R}$ & & & 3187201 \\
\hline Patagioenas cayennensis (Bonnaterre, 1792) & $\mathrm{R}$ & & & 3187201 \\
\hline Zenaida auriculata (Des Murs, 1847) & $\mathrm{R}$ & & & 3186127 \\
\hline Leptotila verreauxi Bonaparte, 1855 & $\mathrm{R}$ & & $\mathrm{N}$ & 3186124 \\
\hline Leptotila rufaxilla (Richard \& Bernard, 1792) & 0 & & & 3198854 \\
\hline \multicolumn{5}{|l|}{ Cuculidae } \\
\hline Coccyzus melacoryphus Vieillot, 1817 & Ra & & & 782876 \\
\hline Guira guira (Gmelin, 1788) & 0 & & & 3189171 \\
\hline Piaya cayana (Linnaeus, 1766) & Ra & & & \\
\hline \multicolumn{5}{|l|}{ Tytonidae } \\
\hline Tyto furcata (Temminck, 1827) & 0 & & & 3189175 \\
\hline \multicolumn{5}{|l|}{ Strigidae } \\
\hline Megascops choliba (Vieillot, 1817) & $\mathrm{R}$ & & & \\
\hline Megascops sanctaecatarinae (Salvin, 1897) & $\mathrm{R}$ & & & 3185145 \\
\hline Bubo virginianus (Gmelin, 1788) & 0 & & & \\
\hline Asio clamator (Vieillot, 1808) & 0 & & & 3189173 \\
\hline \multicolumn{5}{|l|}{ Caprimulgidae } \\
\hline Lurocalis semitorquatus (Gmelin, 1789) & $\mathrm{R}$ & & & \\
\hline Hydropsalis longirostris (Bonaparte, 1825) & $\mathrm{R}$ & & $P$ & 895226 \\
\hline Hydropsalis torquata (Gmelin, 1789) & 0 & & & 3191668 \\
\hline Podager nacunda (Vieillot, 1817) & Ra & & & \\
\hline Apodidae & & & & \\
\hline Cypseloides senex (Temminck, 1826) & $\mathrm{R}$ & & & 988813 \\
\hline Streptoprocne zonaris (Shaw, 1796) & $\mathrm{R}$ & & & 938730 \\
\hline Streptoprocne biscutata (Sclater, 1866) & $\mathrm{R}$ & & & 2799903 \\
\hline Chaetura meridionalis Hellmayr, 1907 & $\mathrm{R}$ & & & \\
\hline Trochilidae & & & & \\
\hline Florisuga fusca (Vieillot, 1817) & 0 & & & 3189169 \\
\hline Stephanoxis loddigesii (Gould, 1831)* & & & & \\
\hline Chlorostilbon lucidus (Shaw, 1812) & $\mathrm{R}$ & & & 3189170 \\
\hline Leucochloris albicollis (Vieillot, 1818) & $\mathrm{R}$ & & & 2805770 \\
\hline Trogonidae & & & & \\
\hline Trogon surrucura Vieillot, 1817 & & & & 3038025 \\
\hline Alcedinidae & & & & \\
\hline Megaceryle torquata (Linnaeus, 1766) & $\mathrm{R}$ & & & 570574 \\
\hline Chloroceryle amazona (Latham, 1790) & $\mathrm{R}$ & & & 766489 \\
\hline Chloroceryle americana (Gmelin, 1788) & $\mathrm{R}$ & & & 766488 \\
\hline Bucconidae & & & & \\
\hline Nystalus chacuru (Vieillot, 1816) & $\mathrm{Ra}$ & & & \\
\hline Picidae & & & & \\
\hline Picumnus nebulosus Sundevall, 1866 & $\mathrm{R}$ & NT (GL) & & \\
\hline Melanerpes candidus (0tto, 1796) & 0 & & & 3198905 \\
\hline Veniliornis spilogaster (Wagler, 1827) & $\mathrm{R}$ & & & \\
\hline Piculus aurulentus (Temminck, 1821) & $\mathrm{R}$ & NT (GL) & & 3191670 \\
\hline Colaptes melanochloros (Gmelin, 1788) & $\mathrm{R}$ & & & \\
\hline Colaptes campestris (Vieillot, 1818) & $\mathrm{R}$ & & & 3185147 \\
\hline Cariamidae & & & & \\
\hline Cariama cristata (Linnaeus, 1766) & $\mathrm{R}$ & & & 3187247 \\
\hline Falconidae & & & & \\
\hline Caracara plancus (Miller, 1777) & $\mathrm{R}$ & & $\mathrm{N}, \mathrm{J}$ & 3187207 \\
\hline Milvago chimachima (Vieillot, 1816) & $\mathrm{R}$ & & J & 3185147 \\
\hline Milvago chimango (Vieillot, 1816) & $\mathrm{R}$ & & & 3191691 \\
\hline Micrastur ruficollis (Vieillot, 1817) & 0 & & & \\
\hline Micrastur semitorquatus (Vieillot, 1817) & Ra & & & 3038035 \\
\hline Falco sparverius Linnaeus, 1758 & $\mathrm{R}$ & & $\mathrm{N}, \mathrm{J}$ & 3191669 \\
\hline Falco femoralis Temminck, 1822 & $\mathrm{R}$ & & $\mathrm{J}$ & 3038034 \\
\hline
\end{tabular}




\section{Taxa $\quad$ Status} Conservation Breeding evidence

Voucher number

\section{Psittacidae}

Occurrence in PET

Pyrrhura frontalis (Vieillot, 1817)

Myiopsitta monachus (Boddaert, 1783)

Pionopsitta pileata (Scopoli, 1769)

Pionus maximiliani (Kuhl, 1820)

Amazona pretrei (Temminck, 1830)

\section{$\mathrm{R}$}

Ra

$\mathrm{R}$

Thamnophilidae

Thamnophilus ruficapillus Vieillot, 1816

Thamnophilus caerulescens Vieillot, 1816

\section{Conopophagidae}

Conopophaga lineata (Wied, 1831)

Scytalopus pachecoi Maurício, 2005

\section{Dendrocolaptidae}

Sittasomus griseicapillus (Vieillot, 1818)

Lepidocolaptes falcinellus (Cabanis \& Heine, 1859)

Dendrocolaptes platyrostris Spix, 1825

Xiphocolaptes albicollis (Vieillot, 1818)

\section{Furnariidae}

Cinclodes pabsti Sick, 1969

Furnarius rufus (Gmelin, 1788)

Lochmias nematura (Lichtenstein, 1823)

Heliobletus contaminatus Berlepsch, 1885

Syndactyla rufosuperciliata (Lafresnaye, 1832)

Leptasthenura striolata (Pelzeln, 1856)

Leptasthenura setaria (Temminck, 1824)

Phacellodomus striaticollis (d'Orbigny \& Lafresnaye, 1838)

Anumbius annumbi (Vieillot, 1817)

Synallaxis cinerascens Temminck, 1823

Synallaxis spixi Sclater, 1856

Limnoctites rectirostris (Gould, 1839)

Cranioleuca obsoleta (Reichenbach, 1853)

\section{Tityridae}

Pachyramphus viridis (Vieillot, 1816)

Pachyramphus polychopterus (Vieillot, 1818)

Pachyramphus validus (Lichtenstein, 1823)

\section{Rhynchocyclidae}

Phylloscartes ventralis (Temminck, 1824)

Tolmomyias sulphurescens (Spix, 1825)

Tyrannidae

Hirundinea ferruginea (Gmelin, 1788)

Camptostoma obsoletum (Temminck, 1824)

Elaenia parvirostris Pelzeln, 1868

Elaenia mesoleuca (Deppe, 1830)

Elaenia obscura (d'Orbigny \& Lafresnaye, 1837)

Phyllomyias virescens (Temminck, 1824)

Phyllomyias fasciatus (Thunberg, 1822)

Serpophaga nigricans (Vieillot, 1817)

Serpophaga subcristata (Vieillot, 1817)

Myiarchus swainsoni Cabanis \& Heine, 1859

Pitangus sulphuratus (Linnaeus, 1766)

Machetornis rixosa (Vieillot, 1819)

Myiodynastes maculatus (Statius Muller, 1776)

Megarynchus pitangua (Linnaeus, 1766)

Tyrannus melancholicus Vieillot, 1819

Tyrannus savana Vieillot, 1808

Empidonomus varius (Vieillot, 1818)

Myiophobus fasciatus (Statius Muller, 1776)

Pyrocephalus rubinus (Boddaert, 1783)

Lathrotriccus euleri (Cabanis, 1868)

Knipolegus cyanirostris (Vieillot, 1818)

Knipolegus lophotes Boie, 1828

Satrapa icterophrys (Vieillot, 1818)

Xolmis cinereus (Vieillot, 1816)

Xolmis irupero (Vieillot, 1823)

Xolmis dominicanus (Vieillot, 1823)

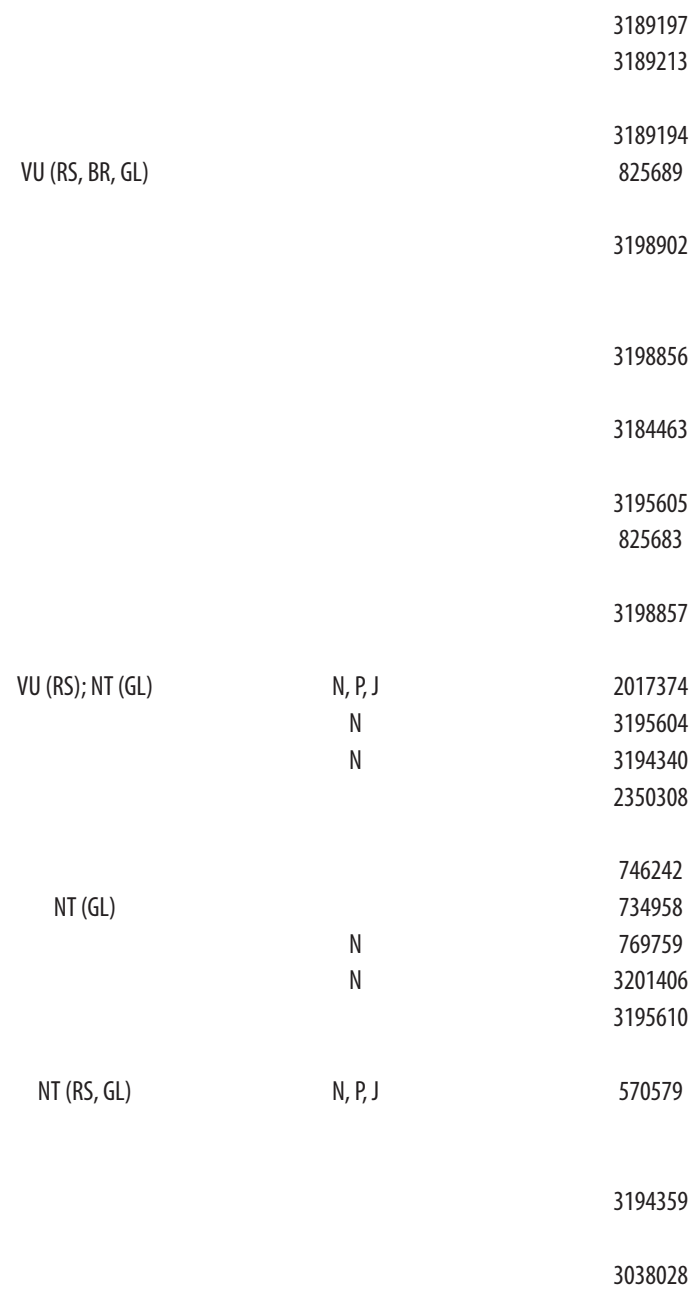

1267851

N

3201405

2366535

766497

2017371

N

3191701

$N, P, J$

3194354

3194345

2017367

782653 


\begin{tabular}{|c|c|c|c|c|}
\hline \multirow{2}{*}{ Taxa } & \multicolumn{2}{|c|}{ Status } & \multirow{2}{*}{ Breeding evidence } & \multirow{2}{*}{ Voucher number } \\
\hline & Occurrence in PET & Conservation & & \\
\hline \multicolumn{5}{|l|}{ Vireonidae } \\
\hline Cyclarhis gujanensis (Gmelin, 1789) & $\mathrm{R}$ & & & \\
\hline Vireo chivi (Vieillot, 1817) & $\mathrm{R}$ & & & 2796089 \\
\hline \multicolumn{5}{|l|}{ Corvidae } \\
\hline Cyanocorax caeruleus (Vieillot, 1818) & $\mathrm{R}$ & NT (GL) & & 3189199 \\
\hline \multicolumn{5}{|l|}{ Hirundinidae } \\
\hline Pygochelidon cyanoleuca (Vieillot, 1817) & $\mathrm{R}$ & & & 3191679 \\
\hline Stelgidopteryx ruficollis (Vieillot, 1817) & $\mathrm{R}$ & & & 3038022 \\
\hline Progne tapera (Vieillot, 1817) & $\mathrm{R}$ & & & 3184464 \\
\hline Progne chalybea (Gmelin, 1789) & Ra & & & 3191680 \\
\hline Tachycineta leucorrhoa (Vieillot, 1817) & $\mathrm{R}$ & & $\mathrm{N}, \mathrm{J}$ & 3191675 \\
\hline Riparia riparia (Linnaeus, 1758) & 0 & & & 3184464 \\
\hline Petrochelidon pyrrhonota (Vieillot, 1817) & 0 & & & 3184486 \\
\hline \multicolumn{5}{|l|}{ Troglodytidae } \\
\hline Troglodytes musculus Naumann, 1823 & $\mathrm{R}$ & & $\mathrm{N}, \mathrm{J}$ & \\
\hline \multicolumn{5}{|l|}{ Turdidae } \\
\hline Turdus leucomelas Vieillot, 1818 & 0 & & & \\
\hline Turdus rufiventris Vieillot, 1818 & $\mathrm{R}$ & & $N, P, J$ & 3195616 \\
\hline Turdus amaurochalinus Cabanis, 1850 & $\mathrm{R}$ & & $P, J$ & \\
\hline Turdus subalaris (Seebohm, 1887) & $\mathrm{R}$ & & & 3195614 \\
\hline Turdus albicollis Vieillot, 1818 & $\mathrm{R}$ & & & \\
\hline \multicolumn{5}{|l|}{ Mimidae } \\
\hline Mimus saturninus (Lichtenstein, 1823) & $\mathrm{R}$ & & $N, P, J$ & 746221 \\
\hline \multicolumn{5}{|l|}{ Motacillidae } \\
\hline Anthus nattereri Sclater, 1878 & $\mathrm{R}$ & VU (RS, BR, GL) & $\mathrm{N}$ & 746219 \\
\hline Anthus hellmayri Hartert, 1909 & $\mathrm{R}$ & & $\mathrm{N}$ & 766490 \\
\hline \multicolumn{5}{|l|}{ Passerelidae } \\
\hline Zonotrichia capensis (Statius Muller, 1776) & $\mathrm{R}$ & & $\mathrm{N}, \mathrm{P}, \mathrm{J}$ & 3197767 \\
\hline Ammodramus humeralis (Bosc, 1792) & $\mathrm{R}$ & & $\mathrm{N}$ & 3197747 \\
\hline Parulidae & & & & \\
\hline Setophaga pitiayumi (Vieillot, 1817) & $\mathrm{R}$ & & & 1211734 \\
\hline Geothlypis aequinoctialis (Gmelin, 1789) & $\mathrm{R}$ & & $N, P$ & 746241 \\
\hline Basileuterus culicivorus (Deppe, 1830) & & & & 3195607 \\
\hline Myiothlypis leucoblephara (Vieillot, 1817) & $\mathrm{R}$ & & & \\
\hline Icteridae & & & & \\
\hline Cacicus chrysopterus (Vigors, 1825) & $\mathrm{R}$ & & $\mathrm{N}$ & \\
\hline Icterus pyrrhopterus (Vieillot, 1819) & Ra & & & \\
\hline Gnorimopsar chopi (Vieillot, 1819) & $\mathrm{R}$ & & & 3194342 \\
\hline Chrysomus ruficapillus (Vieillot, 1819) & Ra & & & \\
\hline Xanthopsar flavus (Gmelin, 1788) & $\mathrm{R}$ & VU (RS, BR, GL) & $N, P, J$ & 1198689 \\
\hline Pseudoleistes guirahuro (Vieillot, 1819) & $\mathrm{R}$ & & $N, P, J$ & 3201402 \\
\hline Agelaioides badius (Vieillot, 1819) & $\mathrm{R}$ & & & 3197748 \\
\hline Molothrus rufoaxillaris Cassin, 1866 & 0 & & & \\
\hline Molothrus bonariensis (Gmelin, 1789) & $\mathrm{R}$ & & & 3194344 \\
\hline Sturnella superciliaris (Bonaparte, 1850) & 0 & & & 3184505 \\
\hline Thraupidae & & & & \\
\hline Pipraeidea melanonota (Vieillot, 1819) & $\mathrm{R}$ & & & 769758 \\
\hline Pipraeidea bonariensis (Gmelin, 1789) & $\mathrm{R}$ & & & 3194357 \\
\hline Stephanophorus diadematus (Temminck, 1823) & $\mathrm{R}$ & & $\mathrm{N}, \mathrm{J}$ & 714484 \\
\hline Tangara sayaca (Linnaeus, 1766) & $\mathrm{R}$ & & & 3194355 \\
\hline Tangara preciosa (Cabanis, 1850) & $\mathrm{R}$ & & & 876238 \\
\hline Sicalis flaveola (Linnaeus, 1766) & $\mathrm{R}$ & & $\mathrm{N}, \mathrm{J}$ & 3195619 \\
\hline Sicalis luteola (Sparrman, 1789) & $\mathrm{R}$ & & $\mathrm{N}, \mathrm{J}$ & 1198694 \\
\hline Haplospiza unicolor Cabanis, 1851 & 0 & & & 876237 \\
\hline Sporophila beltoni Repenning \& Fontana, 2013 & 0 & EN (RS); VU (BR) & $\mathrm{N}$ & 3197762 \\
\hline Sporophila caerulescens (Vieillot, 1823) & $\mathrm{R}$ & & $\mathrm{N}, \mathrm{J}$ & 3195621 \\
\hline Sporophila hypoxantha Cabanis, 1851 & 0 & VU (RS, BR) & & 825687 \\
\hline Sporophila melanogaster (Pelzeln, 1870) & $\mathrm{R}$ & EN (RS); VU (BR); NT (GL) & $N, P, J$ & 2484591 \\
\hline Embernagra platensis (Gmelin, 1789) & $\mathrm{R}$ & & $N, P, J$ & 2017375 \\
\hline Emberizoides ypiranganus Ihering \& Ihering, 1907 & $\mathrm{R}$ & & $\mathrm{N}, \mathrm{P}, \mathrm{J}$ & 1862870 \\
\hline Saltator similis d'Orbigny \& Lafresnaye, 1837 & $\mathrm{R}$ & & & \\
\hline Saltator maxillosus Cabanis, 1851 & 0 & & & 3195603 \\
\hline Poospiza nigrorufa (d'Orbigny \& Lafresnaye, 1837) & $\mathrm{R}$ & & $P$ & 3194358 \\
\hline Microspingus cabanisi Bonaparte, 1850 & $\mathrm{R}$ & & & \\
\hline Donacospiza albifrons (Vieillot, 1817) & $\mathrm{R}$ & & $\mathrm{N}, \mathrm{J}$ & 714432 \\
\hline Fringillidae & & & & \\
\hline Spinus magellanicus (Vieillot, 1805) & $\mathrm{R}$ & & J & 3195617 \\
\hline
\end{tabular}


season. In the season 2016-2017 three young males were recorded in different marshes of PET and one adult female had a successful nest (see details about the nest in breeding data).

Mesembrinibis cayennensis (Near Threatened - RS): found along the riparian forest of the Tainhas river, usually in small groups (four to six individuals). Often detected by its voice when flying over the river near dawn and dusk.

Sarcoramphus papa (Near Threatened - RS): not recorded in our survey. The occurrence of this species for the PET is mentioned only in the management plan of the park.

Geranoaetus melanoleucus (Near Threatened - RS): sighted with less frequency than the Crowned Eagle (Urubitinga coronata). The records are probably of a few individuals, which suggests low density in the park.

Picumnus nebulosus (Near Threatened - GL): this small woodpecker is found in riparian forests and small forest patches around marshes. It can eventually be seen inside marshes crossing from one forest patch to another.

Piculus aurulentus (NearThreatened - GL): found with frequency in the park, usually in riparian forests.

Leptasthenura setaria (Near Threatened - GL): associated with araucaria forests (Araucaria angustifolia), easily found in the park, usually in pairs.

Limnoctites rectirostris (Near Threatened - RS, GL): it was recorded only in marshes with "gravatá" (Eryngium spp., mainly E. pandanifolium). Although common, it occurs in low densities in the PET.

Cyanocorax caeruleus (Near Threatened - GL): this species is easily heard or sighted in the PET, moving along forest areas.

\section{Breeding data}

We determined at least 55 species breeding in the PET or in its vicinities. Only one of these species do not have regular occurrence in the park (S. beltoni is occasional) (Table 1). Data on the reproduction of some species are given below.

Rhynchotus rufescens: one nest containing nine eggs was found on January $2^{\text {nd }}, 2013$. The nest was in a dry grassland area under a little clump of grass.

Nothura maculosa: one nest with seven eggs was found on December $3^{\text {rd }}, 2013$ in a dry grassland area.

Urubitinga coronata: a pair was often sighted accompanied by a juvenile in 2012-2013. On December $2^{\text {nd }}, 2012$ a juvenile was seen together with an adult, which was carrying a snake in flight. A photo taken on January $13^{\text {th }}, 2013$ shows this juvenile molting the first primaries (P1 to P4), indicating it was a little more than one year old individual. In the following season, only a juvenile was observed in the park. In 2016-2017 another juvenile (differentiated by its plumage) was recorded together with an adult. In 2017-2018 probably the same juvenile and adult individuals were observed several times. All records occurred in an area of approximately 400 ha inside the PET's boundaries.

Geranoaetus melanoleucus: juvenile individuals were observed in two occasions together with adults in late 2012. Because the time-span between both records is relatively short, they may be the same individuals.

Gallinago paraguaiae: one nest was found in a marsh on October $23^{\text {rd }}, 2017$ containing three eggs. The nest was built in a small elevation inside the marsh among grasses.

Hydropsalis longirostris: two fledglings were found in a rocky outcrop in a hill slope on December $26^{\text {th }}, 2015$. An adult was nearby and tried to distract the observer with short and erratic flights.

Cinclodes pabsti: nest activity of this species was observed in all seasons at the headquarters of PET. The species built the nest in the gap between the roof tiles, which formed a cavity. In 2017 a pair successfully raised two broods in the same season, one in September and another in December.

Lochmias nematura: one nest was found in a slope on the banks of the Tainhas river on September $25^{\text {th }}, 2014$, in a visit made in the PET outside the sampling period. An adult was seen carrying food on constant visits to the nest. At the time of the observations the nest was about $30 \mathrm{~cm}$ above the water level.

Phacellodomus striaticollis: some nests built in small trees in the marsh edge were found in the park. The activity of one nest was verified by the presence of adults carrying food on January $10^{\text {th }}, 2017$.

Limnoctites rectirostris: several active nests of this species were found and monitored in the PET, all located in marshes with Eryngium spp.

Xolmis dominicanus: three fledglings being fed by parents were observed in a marsh on January $1^{\text {st }}, 2013$. In November 2017 adults were sighted carrying food, but the nest was not found.

Anthus hellmayri: one nest with two eggs was found on November $21^{\text {st }}, 2016$ in a dry grassland area with low grasses. The nest was positively identified by the adult which left it.

Anthus nattereri: one nest with two nestlings was found on November $9^{\text {th }}, 2017$ in a dry grassland area under a low clump of grasses. The nest was on the top of a hill, where A. hellmayri congener was not recorded. 
Geothlypis aequinoctialis: one nest with two nestlings and one egg was found in a marsh on November $20^{\text {th }}$, 2013 , built in a clump of grasses.

Xanthopsar flavus: a nesting colony with approximately 40 individuals was found in a marsh surrounded by dry grassland with low grasses on November $25^{\text {th }}, 2013$. The nests were in the center of the marsh, and two of them contained four nestlings each.

Pseudoleistes guirahuro: two juveniles being fed by adults were observed in a marsh on January $5^{\text {th }}, 2016$. In early February 2018 adults were sighted carrying food, but the nest was not found.

Donacospiza albifrons: adults carrying material to build a nest were observed on_December $20^{\text {th }}, 2012$, and fledglings being fed by parents were observed on January $15^{\text {th }}, 2016$.

Sicalis luteola: one nest found on November 21 1 st 2013 contained five eggs and was built in a site with clumps of tall and dry grasses.

Emberizoides ypiranganus: more than 200 nests of this species were monitored during the studied seasons. The species uses both wet and dry areas to build nests, always in clumps of grasses. The use of dry grasslands to breed varies according to grassland management and time since last burn. In sites periodically burned the nests tend to be built in marshes more than in dry areas.

Embernagra platensis: approximately 50 nests were found and monitored in the PET. Nests were active from early October to early February. The species usually breeds in wet areas or close to them, often using large clumps to build its nests.

Sporophila melanogaster: more than 60 nests of this threatened species were monitored in the PET. Nests were built both in marshes and dry grasslands from late November to early March. Small shrubs and grass clumps are used to build its nests.

Sporophila beltoni: one nest with two eggs was found on February $17^{\text {th }}, 2017$, in a marsh $2 \mathrm{~km}$ far from the Tainhas river, in the west border of PET. The marsh is dominated by Eryngium pandanifolium and Ludwigia sericea in plain terrain. During the entire nesting period only the female was seen attending the nest, and on March $8^{\text {th }}, 2017$ two fledglings left the nest.

\section{DISCUSSION}

\section{Species list}

Our results show the occurrence of 76 species not previously mentioned for the Parque Estadual do Tainhas, representing an increase of $58 \%$ in the bird richness known to this protected area. The larger richness found in this study is mainly associated with the greater sample effort, since in the single previous survey performed at the park (management plan, Bencke \& Duarte, 2008) there were only few days of fieldwork. The data compilation shows that the PET has, at least, 208 bird species, including 146 that can regularly be found in the park. The core avifauna (i.e., regular species) is of greater interest to the community ecology or conservation of an area (Remsen, 1994).

This total represents approximately $30 \%$ of the bird richness known to Rio Grande do Sul state (Franz et al., 2018) and 61\% of the birds recorded in Campos de Cima da Serra region (Fontana et al., 2008a). In relation to other protected areas in the region, the PET presents $69 \%$ of the bird richness recorded in Parque Nacional de Aparados da Serra and Parque Nacional da Serra Geral (IBAMA, 2004). However, the greater richness in these two protected areas can be attributed to the presence of an altitudinal gradient with several phytophysiognomies and species exclusive of some habitats, besides the larger area of these parks.

Three species previously reported for PET that were not recorded in our study are water-related species (P. podiceps, P. melanops, and J. jacana), and other two are forest-related species (S. papa and S. loddigesii). The lack of these forest species during our fieldwork may be associated with the subsampling of the large araucaria forest patches found inside the park, such as those at the northern limit of PET: five forest species were exclusively recorded there (Trogon surrucura, Synallaxis cinerascens, Pachyramphus validus, Tolmomyias sulphurescens, and Basileuterus culicivorus). It is possible that further sampling concentrated on forested areas of the park increases species richness even more.

Some species here classified as regular are migratory species in Rio Grande do Sul state, which are found in the south of Brazil only during spring and summer seasons (from September to March). Most of these species are known as summer residents, which come to Rio Grande do Sul to breed during the hottest months, such as Elaenia parvirostris, E. mesoleuca, Myiarchus swainsonii, Tyrannus savana, Tyrannus melancholicus, and Vireo chivi (Bencke, 2001; Belton, 2003). Other migrants are visitors from the Northern Hemisphere, and their occurrence in the PET is occasional (e.g., Actitis macularius, Riparia riparia, and Petrochelidon pyrrhonota). These Northern Hemisphere migrants probably use the area only for short periods during their migration. Although our study did not sample birds on cold seasons (winter and fall), this limitation does not seem to have biased the results, since Southern Hemisphere migrants, which visit Rio Grande do Sul state in the winter (sensu Bencke, 2001), are not reported to Campos de Cima da Serra region (Belton, 2003; Fontana et al., 2008a, b; Repenning et al., 2010).

The species classified as locally rare (observed only once) or occasional were represented by lone individuals mostly. These individuals were probably passing through the area, using it only temporarily in their displacements, as observed with Chauna torquata (two 
individuals passed over the park on October $14^{\text {th }}, 2017$, in south-north direction), Columbina talpacoti (a lone male was observed near the headquarters of the park on February $\left.1^{\text {st }}, 2017\right)$, Myiopsitta monachus (five individuals were in a peach tree around the headquarters on March $5^{\text {th }}, 2018$ ), Xolmis irupero (a lone individual was observed only on January $4^{\text {th }}, 2014$ and December 20th 2017 ), Riparia riparia (a lone individual together with a flock of Petrochelidon pyrrhonota, Progne tapera and Tachycineta leucorrhoa was observed on October 29th, 2016 and again on October 22 ${ }^{\text {nd }}, 2017$ ), and Sturnella superciliaris (a lone male was observed only on December 13th, 2016 and November $\left.13^{\text {th }}, 2017\right)$. Some of them were recorded in two different years but in a similar time of the year, probably the period in which the species passes through the area of the park. According to Remsen (1994), in a survey, the number of occasional, wandering or dispersing species from other habitats or regions (i.e., individuals not characteristic of that habitat) depends more on the observer effort and may not reach an asymptote within the time limits of most studies.

In this group of rare and occasional species in the PET we can highlight some that are quite conspicuous and common in other regions of Rio Grande do Sul, such as Plegadis chihi, Phimosus infuscatus, Rostrhamus sociabilis, C. talpacoti, Piaya cayana, M. monachus, X. irupero, Progne chalybea, Chrysomus ruficapillus, and S. superciliaris (Belton, 2003). Many of these species have few records in Campos de Cima da Serra region and some of them probably indicate cases of recent colonization and may be associated with habitat transformation (Fontana et al., 2008a; Repenning et al., 2010). On the other hand, several uncommon species in Rio Grande do Sul are quite common in the PET, such as Cinclodes pabsti, $X$. dominicanus, X. flavus, A. nattereri, and S. melanogaster. They are threatened species regularly found in the park and some have a considerable population locally.

We emphasize the great number of birds of prey (vultures, hawks, falcons and owls) found during our fieldwork ( $n=26$ species, $13 \%$ of the bird richness recorded in the period). This shows the good conservation status of the area, since these species are indicators of environmental quality and are associated with habitats with greater biodiversity (Sergio et al., 2005, 2006). Among these species we can highlight the regular presence of the Crowned Eagle (U. coronata) inside the PET. Campos de Cima da Serra are the only region in Rio Grande do Sul state where this large eagle has been seen regularly over the past few decades (Bencke \& Duarte, 2008). Others are less frequent and even rare in the area, such as Circus buffoni, Geranospiza caerulescens, R. sociabilis, Urubitinga urubitinga, Accipiter bicolor, and Micrastur semitorquatus.

\section{Distributional novelties}

Records of C. torquata, Melanerpes candidus, G. caerulescens, Megarynchus pitangua, and R. riparia represent novelties to the avifauna of Campos de Cima da Serra, since these species were not reported in local (e.g.,
Fontana et al., 2008a, b; Repenning et al., 2010) and regional works (e.g., Belton, 2003). Other records can be considered as the limits of distribution of the species or small range extensions. The record of the Tropeiro Seedeater (S. beltoni) corresponds to the southernmost point of its distribution, $20 \mathrm{~km}$ far from the previous southernmost record (Repenning \& Fontana, 2013). The record of the Planalto Tapaculo (Scytalopus pachecoi) probably corresponds to the southernmost limit of distribution of the population that occurs in the highlands of the Meridional Plateau. This species occurs in three disjoint areas in Rio Grande do Sul (Maurício, 2005). Inside the PET, the species probably occurs only in the north of "Passo do S" waterfall, where it was recorded in the riparian forest of a tributary on the left margin of the Tainhas river.

\section{Breeding}

The total of 55 species breeding in the PET or in its vicinities (being 54 regulars in the park), including threatened and/or migratory grassland species, emphasizes the importance of this protected area. Although it was not possible to find evidence of breeding for 92 regular species, we believe that the sedentary species use the area to breed. For sedentary species, the occurrence within the region (in the breeding season or not), and the presence of proper breeding habitats are regarded in the scale (at the low categories) for breeding evidence proposed by Binford (1973). The absence of breeding data on these species may reflect the difficulty to find evidences of reproduction (nests or fledglings) and highlights the necessity for further specific studies in the area.

The area houses a considerable breeding population of Black-bellied Seedeater (S. melanogaster) from November to March. Campos de Cima da Serra region is extremely important to this species, which has its breeding area limited to highland grasslands of Rio Grande do Sul and Santa Catarina (Bencke et al., 2003; Rovedder \& Fontana, 2012). Moreover, the PET and its surroundings serve as an important breeding area for one of the largest birds of prey in Brazil, the Crowned Eagle (U. coronata). Although we did not find nests of this species, the recurring records of juveniles inside the park's boundaries show that the area can be important, at least, providing food for growth of immature birds. It is believed that juveniles remain with adults for long periods or even years (Ferguson-Lees \& Christie, 2001). Armadillos (mostly Dasypus spp.) and snakes are apparently a significant item in the diet of U. coronata (see Ferguson-Lees \& Christie, 2001; Bierregaard et al., 2019). Our observations of individuals feeding on these items and the apparent high abundance of armadillos in the area, associated with the several records of the Crowned Eagle (adults and juveniles) in a relatively small area of PET, suggest that the species often uses the park to forage.

Some grassland species in the PET use specific habitats for breeding, such as Straight-billed Reedhaunter (L. rectirostris), which breeds only in marshes with "gravatás" (Eryngium spp.), using mainly E. pandanifolium to 
build its nests (Larre, 2017). Other grassland species may use both wet (marshes and wet grasslands) and dry areas as nest sites, such as E. ypiranganus and S. melanogaster (Rovedder \& Fontana, 2012; Chiarani \& Fontana, 2015). The nest site of the Tropeiro Seedeater can be considered atypical for the species, since it uses high shrubs in rugged and steep relief to breed (Repenning \& Fontana, in press), and we found a nest in a marsh dominated by E. pandanifolium and Ludwigia sericea in plain terrain.

Breeding evidences of two species reported in our study (Lochmias nematura and A. nattereri) are not presented in the broad review on the breeding status of birds in Rio Grande do Sul, made by Maurício et al. (2013). However, our records could be accepted as effective breeding evidences according to the criteria established in Maurício et al. (2013) and could include these species in the list of bird species that breed in Rio Grande do Sul state. Besides, Belton (2003) had considered the evidences of breeding for these two species reasonable, because he accepted other breeding evidences (e.g., enlarged gonads, nests under construction, and some display behaviors), which were considered as indirect evidences and were not accepted in Maurício et al. (2013).

\section{CONCLUSIONS}

Our results extend the knowledge on the birds that occur in Parque Estadual do Tainhas. Of the 18 species of conservation concern recorded in the PET (ten threatened and eight near-threatened), 15 of them use the area of the park regularly and, at least, nine use the park or its vicinities to breed, such as U. coronata, C. pabsti, $L$. rectirostris, $X$. dominicanus, $A$. nattereri, $X$. flavus, and S. melanogaster. The number and composition of species recorded in the PET reinforce the importance of this protected area for bird conservation, mainly those grassland-dependent species, supporting the indication of the region as an Important Bird Area.

Since vast areas along the Tainhas river have already been completely forested by commercial plantations of Pinus spp. (Bencke et al., 2006), appropriate protection and management measures, such as the acquisition of all areas of the park, are fundamental for the long-term bird conservation in the PET as well as in Campos de Cima da Serra.

\section{ACKNOWLEDGEMENTS}

We thank Christian B. Andretti and Gabriel G. Larre for assistance in some field surveys. Glayson A. Bencke first for providing information on the previous survey made in Parque Estadual do Tainhas and on specimens deposited in Museu de Ciências Naturais. Secondly for helping to determine the age of one of the Crowned Eagle juveniles recorded. The manuscript was greatly improved with comments and suggestions by Luís $F$. Silveira and anonymous referees. We thank Patrícia B. Schafer for improving the English writing of the manu- script. Administrative team of PET for the support with logistics and permission to use the field base in the study area. Landowners that allowed access to their private areas in the park. Instituto Chico Mendes de Conservação da Biodiversidade (ICMBio) and Secretaria do Meio Ambiente e Desenvolvimento Sustentável (SEMA) for research permission. EC received a scholarship from Conselho Nacional do Desenvolvimento Científico e Tecnológico (CNPq) (process 141055/2015-4). Ministério da Ciência, Tecnologia, Inovações e Comunicações (MCTIC) through CNPq grants to CSF (processes 457475/2012-9, 303318/2013-6, 309438/2016-0, and 402083/2016-4). Neotropical Grassland Conservancy also funded part of this research.

\section{REFERENCES}

Almeida, J.A. 2009. Fatores abióticos. In: Boldrini, I.I. (Ed.). Biodiversidade dos campos do Planalto das Araucárias. Brasília, Ministério do Meio Ambiente. p. 20-25.

Andrade, B.; Bonilha, C.L.;Overbeck, G.; Vélez-Martin, E.; Rolim, R.; Bordignon, S.A.L.; Schneider, A.A.; Ely, C.V.; Lucas, D.; Garcia, É.N.; dos Santos, E.D.; Torchelsen, F.P.; Vieira, M.; Filho, P.J.J.S.; Ferreira, P.M.A.; Trevisan, R.; Hollas, R.; Campestrini, S.; Pillar, V.D. \& Boldrini, I.I. 2019. Classification of South Brazilian grasslands: implications for conservation. Applied Vegetation Science, 22: 168-184.

Azpiroz, A.B.; Isacch, J.P.; Dias, R.A.; Di Giacomo, S.A.; Fontana, C.S. \& Palarea, C.M. 2012. Ecology and conservation of grassland birds in southeastern South America: A review. Journal of Field Ornithology, 83: 217-246.

Belton, W. 2003. Aves do Rio Grande do Sul: distribuição e biologia. São Leopoldo, Editora Unisinos.

Bencke, G.A. 2001. Lista de referência das aves do Rio Grande do Sul. Porto Alegre, Fundação Zoobotanica do Rio Grande do Sul. 102p.

Bencke, G.A. \& Duarte, M.M. 2008. Projeto conservação da Mata Atlântica no Rio Grande do Sul: plano de manejo do Parque Estadual do Tainhas. Porto Alegre, Secretaria de Estado do Meio Ambiente do Rio Grande do Sul.

Bencke, G.A.; Fontana, C.S.; Dias, R.A.; Maurício, G.N. \& Mähler Jr., J.K.F. 2003. Aves. In: Fontana, C.S.; Bencke, G.A. \& Reis, R.E. (Orgs.). Livro vermelho da fauna ameaçada de extinção no Rio Grande do Sul. Porto Alegre, Edipucrs. p. $189-479$.

Bencke, G.A.; Maurício, G.N.; Develey, P.F. \& Goerck, J.M. (Orgs.). 2006. Áreas Prioritárias para a Conservação de aves no Brasil. Parte I - estados do domínio da Mata Atlântica. São Paulo, SAVE Brasil.

Bierregaard, R.0.; Christie, D.A.; Kirwan, G.M. \& Marks, J.S. 2019. Crowned Solitary Eagle (Buteogallus coronatus). In: del Hoyo, J.; Elliott, A.; Sargatal, J.; Christie, D.A. \& de Juana, E. (Eds.). Handbook of the Birds of the World Alive. Barcelona, Lynx Edicions.

Binford, L.C. 1973. A short method for treating avian breeding data in regional accounts. Wilson Bulletin, 85(2): 244-246.

Boldrini, I.I. 2009. A flora dos campos do Rio Grande do Sul. In: Pillar, V.D.; Müller, S.C.; Castilhos, Z.M.S. \& Jacques, A.V.A. (Eds.). Campos sulinos: conservação e uso sustentável da biodiversidade. Brasília, Ministério do Meio Ambiente. p. 63-77.

Bond, W.J. \& Parr, C.L. 2010. Beyond the forest edge: Ecology, diversity and conservation of the grassy biomes. Biological Conservation, 143: 2395-2404.

Bond-Buckup, G. \& Dreier, C. 2008. Paisagem natural: desvendando a região. In: Bond-Buckup, G. (Ed.). Biodiversidade dos Campos de Cima da Serra. Porto Alegre, Libretos. p. 11-17. 
Buckup, L. \& Bond-Buckup, G. 2008. Ameaças: impactos na natureza. In: Bond-Buckup, G. (Ed.). Biodiversidade dos Campos de Cima da Serra. Porto Alegre, Libretos. p. 149-153.

Chiarani, E. \& Fontana, C.S. 2015. Breeding biology of the Lesser Grass-Finch (Emberizoides ypiranganus) in southern Brazilian upland grasslands. The Wilson Journal of Ornithology, 127(3): 441-456.

Colwell, R.K. 2013. EstimateS: Statistical Estimation of Species Richness and Shared Species from Samples. Version 9.1.0. Available at: http://purl.oclc. org/estimates. Access in: 14/03/2019.

Ferguson-Lees, J. \& Christie, D.A. 2001. Raptors of the World. New York, Houghton Mifflin Company.

Fontana, C.S.; Bencke, G.A. \& Reis, R.E. 2003. Livro vermelho da fauna ameaçada de extinção no Rio Grande do Sul. Porto Alegre, Edipucrs.

Fontana, C.S.; Rovedder, C.E.; Repenning, M. \& Gonçalves, M.L. 2008a. Estado atual do conhecimento e conservação da avifauna dos Campos de Cima da Serra do sul do Brasil, Rio Grande do Sul e Santa Catarina. Revista Brasileira de Ornitologia, 16(4): 281-307.

Fontana, C.S.; Repenning, M.; Rovedder, C.E. \& Gonçalves, M.L. 2008b. Aves. In: Bond-Buckup, G. (Ed.). Biodiversidade dos Campos de Cima da Serra. Porto Alegre, Libretos. p. 118-135.

Franz, I.; Agne, C.E.; Bencke, G.A.; Bugoni, L. \& Dias, R.A. 2018. Four decades after Belton: a review of records and evidences on the avifauna of Rio Grande do Sul, Brazil. Iheringia. Série Zoologia, 108: e2018005. DOI

Freitas, G.H.S.; Costa, L.M.; Rodrigues, M. \& Ferreira, M.J.D. 2008. The range of Long-tailed Cinclodes Cinclodes pabsti. Bulletin of the British Ornithologists' Club, 128: 215-216.

Gibson, D.J. 2009. Grasses and Grassland Ecology. New York, Oxford University Press.

Gotelli, N.J. \& Colwell, R.K. 2010. Estimating species richness. In: Magurran, A.E. \& McGill, B.J. (Eds.). Biological diversity: frontiers in measurement and assessment. Oxford, 0xford University Press. p. 39-54.

Hasenack, H. \& Lucatelli, L.M.L. 2008. Ambientes da região: principais feições da paisagem. In: Bond-Buckup, G. (Ed.). Biodiversidade dos Campos de Cima da Serra. Porto Alegre, Libretos. p. 25.

Instituto Brasileiro de Geografia e Estatística (IBGE). 2004. Mapa da vegetação do Brasil e Mapa de Biomas do Brasil. Brasília, IBGE. Available at: http:// www.ibge.gov.br. Access in: 15/09/2018.

Instituto Brasileiro do Meio Ambiente e dos Recursos Naturais Renováveis (IBAMA). 2004. Plano de Manejo dos Parques Nacional de Aparados da Serra e Serra Geral. Brasília. Ministério do Meio Ambiente, dos Recursos Hídricos e da Amazônia Legal.

International Union for Conservation of Nature and Natural Resources (IUCN). 2018. The IUCN Red List of Threatened Species. Version 2018-2. Available at: http://www.iucnredlist.org. Access in: 01/12/2018.

Larre, G.G. 2017. História de vida de Limnoctites rectirostris (Aves: Furnariidae) nos Campos de Cima da Serra, sul do Brasil. (Masters Dissertation). Porto Alegre, Pontifícia Universidade Católica do Rio Grande do Sul.

Maluf, J.R.T. 1999. Nova classificação climática do Rio Grande do Sul. Passo Fundo, Embrapa Trigo. Pesquisa em Andamento Online, 8. Available at: http:// www.cnpt.embrapa.br/biblio/p pa08.htm. Access in: 15/09/2018.

Maurício, G.N. 2005. Taxonomy of southern populations in the Scytalopus speluncae group, with description of a new species and remarks on the systematics and biogeography of the complex (Passeriformes: Rhinocryptidae). Ararajuba, 13(1): 7-28.

Maurício, G.N.; Bencke, G.A.; Repenning, M.; Machado, D.B.; Dias, R.A. \& Bugoni, L. 2013. Review of the breeding status of birds in Rio Grande do Sul, Brazil. Iheringia. Série Zoologia, 103: 163-184.

Ministério do Meio Ambiente (MMA). 2000. Avaliação e ações prioritárias para a conservação da biodiversidade da Mata Atlântica e Campos Sulinos. Brasília, Secretaria de Biodiversidade e Florestas. 40p.
Ministério do Meio Ambiente (MMA). 2014. Lista nacional oficial de espécies da fauna ameaçadas de extinção - Portaria MMA № 444 de 17 de dezembro de 2014. Diário Oficial da União, Brasília, 245: 121-126. Available at: http://www.icmbio.gov.br/portal/images/stories/biodiversidade/ fauna-brasileira/avaliacao-do-risco/PORTARIA N\%C2\%BA 444 DE 17 DE DEZEMBRO DE 2014.pdf.

Overbeck, G.E.; Muller, S.C.; Fidelis, A.; Pfadenhauer, J.; Pillar, V.D.; Blanco, C.C.; Boldrini, I.I.; Both, R. \& Forneck, E.D. 2007. Brazil's neglected biome: the South Brazilian Campos. Perspectives in Plant Ecology, Evolution and Systematics, 9: 101-116.

Overbeck, G.E.; Vélez-Martin, E.; Scarano, F.R.; Lewinsohn, T.M.; Fonseca, C.R.; Meyer, S.T.; Müller, S.C.; Ceotto, P.; Dadalt, L.; Durigan, G.; Ganade, G. Gossner, M.M.; Guadagnin, D.L.; Lorenzen, K.; Jacobi, C.M.; Weisser, W.W. \& Pillar, V.D. 2015. Conservation in Brazil needs to include non-forest ecosystems. Diversity and Distributions, 21: 1455-1460.

Piacentini, V.Q.; Aleixo, A.; Agne, C.E.; Maurício, G.N.; Pacheco, J.F.; Bravo, G.A.; Brito, G.R.R.; Naka, L.N.; Olmos, F.; Posso, S.; Silveira, L.F.; Betini, G.S.; Carrano, E.; Franz, I.; Lees, A.C.; Lima, L.M.; Pioli, D.; Schunck, F.; Amaral, F.R.; Bencke, G.A.; Cohn-Haft, M.; Figueiredo, L.F.A.; Straube, F.C. \& Cesari, E. 2015. Annotated checklist of the birds of Brazil by the Brazilian Ornithological Records Committee/Lista comentada das aves do Brasil pelo Comitê Brasileiro de Registros Ornitológicos. Revista Brasileira de Ornitologia, 23: 91-298.

Pillar, V.D. \& Velez, E. 2010. Extinction of the southern plains in conservation areas: a natural phenomenon or an ethical problem? Natureza \& Conservação, 8: 84-86.

Rambo, B. 2000. A fisionomia do Rio Grande do Sul: ensaio de monografia natural. 3.ed. São Leopoldo, Editora Unisinos.

Remsen Jr., J.V. 1994. Use and misuse of bird lists in community ecology and conservation. The Auk, 111(1): 225-227.

Repenning, M. \& Fontana, C.S. in press. Nesting information for Tropeiro Seedeater (Sporophila beltoni), an endemic songbird from southern Brazil. Revista Brasileira de Ornitologia.

Repenning, M. \& Fontana, C.S. 2013. A new species of gray seedeater (Emberizidae: Sporophila) from upland grasslands of southern Brazil. The Auk, 130(4): 791-803.

Repenning, M.; Rovedder, C.E. \& Fontana, C.S. 2010. Distribuição e biologia de aves nos campos de altitude do sul do Planalto Meridional Brasileiro. Revista Brasileira de Ornitologia, 18: 283-306.

Rio Grande do Sul. 2014. Lista das espécies da fauna ameaçadas de extinção no estado do Rio Grande do Sul. Instrução Normativa № 3. DECRETO № 51.797. Porto Alegre, Diário Oficial do Estado do Rio Grande do Sul, Secretaria do Meio Ambiente, 8 de setembro de 2014.

Rovedder, C.E. \& Fontana, C.S. 2012. Nest, eggs, and nest placement of the Brazilian endemic Black-bellied Seedeater (Sporophila melanogaster). Wilson Journal of Ornithology, 124(1): 173-176.

Serafini, P.P. (Org.). 2013. Plano de Ação Nacional para a conservação dos passeriformes ameaçados dos Campos Sulinos e Espinilho. Brasília, Instituto Chico Mendes de Conservação da Biodiversidade/ICMBio. 212p. (Série Espécies Ameaçadas, 31)

Sergio, F.; Newton, I. \& Marchesi, L. 2005. Top predators and biodiversity. Nature, 236: 192.

Sergio, F.; Newton, I.; Marchesi, L. \& Pedrini, P. 2006. Ecologically justified charisma: preservation of top predators delivers biodiversity conservation. Journal of Applied Ecology, 43: 1049-1055.

Sick, H. 1997. Ornitologia Brasileira. 2.ed. Rio de Janeiro, Editora Nova Fronteira. Stotz, D.F.; Fitzpatrick, J.W.; Parker III, T.A. \& Moskovits, D.K. 1996. Neotropical birds: ecology and conservation. Chicago, University of Chicago Press.

Vieira, E.F. 1984. Rio Grande do Sul: geografia física e vegetação. Porto Alegre, Editora Sagra. 184p. 